Revue européenne des sciences sociales

European Journal of Social Sciences

XLVIII-145 | 2010

À la recherche des fondements de la rationalité

\title{
Della cultura: storia e teoria
}

\section{Giovanni Busino}

\section{(2) OpenEdition}

\section{Journals}

Edizione digitale

URL: http://journals.openedition.org/ress/740

DOI: $10.4000 /$ ress.740

ISSN: 1663-4446

\section{Editore}

Librairie Droz

\section{Edizione cartacea}

Data di pubblicazione: 1 mars 2010

Paginazione: 63-80

ISBN: 978-2-600-01427-4

ISSN: 0048-8046

Notizia bibliografica digitale

Giovanni Busino, « Della cultura: storia e teoria », Revue européenne des sciences sociales [En ligne], XLVIII-145 | 2010, mis en ligne le 01 mars 2013, consulté le 10 décembre 2020. URL : http:// journals.openedition.org/ress/740; DOI : https://doi.org/10.4000/ress.740 


\section{Giovanni BUSINO}

\section{DELLA CULTURA : STORIA E TEORIA}

\section{DEGLI USI DELLA NOZIONE}

Nozione importante benché ambigua e confusa, polisemica, polivalente, purtroppo correntemente utilizzata anche nel linguaggio ordinario (sc. cultura della morte, cultura dell'odio, cultura aziendale, cultura dei migranti, cultura originale, cultura dei giovani, ecc.), oggi, nelle scienze dell'uomo e della società, è divenuta quasi sinonimo di civiltà, colla quale talvolta è fusa o confusa. La storiografia ne appalesa però, la funzione ideologica avuta nei dibattiti intellettuali (universalismo, particolarismo, relativismo, differenzialismo, decadenza della civiltà, fine della cultura, difesa della cultura, progresso, ruolo dell'Occidente, fine della storia, ecc.) e l'importante ruolo esercitato nella critica dell'etnocentrismo e dell'evoluzionismo, giudicati responsabili d'ignorare o di sottovalutare le diversità culturali, di gerarchizzare le società, di trattare le forme e gli stati delle società come se fossero stadi dello sviluppo economico. Il paradigma culturalista ha fornito alle teorie ed alle ricerche una rappresentazione ideologica della società, la quale, nel corso degli anni, è stata estesa dall'ambiente fisico e sociale ai criteri per definire l'uomo stesso, criteri facenti astrazione delle caratteristiche psicologiche panculturali invarianti. Rimandando a ciò che è creato e trasmesso dall'uomo, ai suoi comportamenti, alle maniere di pensare, di sentire, d'agire, più o meno formalizzati, a ciò che non è dato dalla natura, dall'ereditarietà, da attributi panumani costanti, dalle strutture, dalle tecniche, dalle istituzioni, dalle norme, dai valori, dai miti, dalle ideologie, la nozione è servita altresì a refutare quella di natura umana universale, a denunciare i sogni del razionalismo, del metodo universale, del linguaggio perfetto, del sistema unitario della natura. Anche quando è stata depurata da tutte le sfumature normative e utilizzata unicamente in maniera descrittiva, questa nozione ha valorizzato una forma di determinismo secondo il quale l'individuo interiorizza la cultura e agisce conformemente ai suoi modelli normativi. Tra l'individuo e la sua cultura d'appartenenza esisterebbe, ovviamente, una certa armonia mentre i conflitti sarebbero all'origine soprattutto di devianze. Perciò la struttura della personalità dipende dalla cultura specifica d'una società e dal sistema dei valori dominanti e modali della stessa. Ogni società è allora una totalità culturale originale. Società simili dal punto di vista economico possono essere diverse dal punto di vista culturale. La cultura diventa così un insieme d'elementi coerenti e complementari. I giudizi, le valutazioni e le percezioni sono determinati dal sistema culturale d'appartenenza. Anche la conoscenza ordinaria è all'unisono col contesto culturale, al contrario 
della conoscenza scientifica prodotta dalla ragione. Persino chi (per es., Archer 1988) ammette che nuovi elementi possano entrare a farne parte e rendere obsoleti i vecchi elementi e sostiene essere le azioni degli uomini i fattori produttivi, proclama tuttavia che il sistema culturale, divenuto autonomo, esercita un'influenza determinante su tutti i livelli socio-culturali e su tutte le successive generazioni d'individui.

Della nozione Kroeber et Kluckhohn (1952) hanno elencato 163 definizioni raggruppate poi in sei categorie (descrittiva, storica, normativa, psicologica, genetica, strutturale). Dal canto suo, Murdock (1949) ha catalogato in «Human Relations Area Files (HRAF)» i tratti di 250 culture ritenute separate tra di loro benché supponga che delle analisi comparative potrebbero mettere in luce analogie spiegabili mediante categorie universali.

Dagli studi sulla cultura sono state alimentate altresì, le dottrine sull'obbligo, per il pensiero occidentale, di rinunciare all'idea d'una umanità unica, di riconoscere il diritto alla differenza ed all'alterità. Insomma, la nozione, fondatrice del determinismo culturale, della visione relativista della società, delle concezioni dell'uguaglianza assiologica, dell'incomparabilità ed incommensurabilità delle culture, è stata utilizzata non soltanto per combattere il razzismo e le dottrine della mentalità primitiva ma altresì per contestare e ricusare i giudizi di valori sulla gerarchia delle società nonché l'universalismo ed il razionalismo dell'Occidente.

I tentativi (Lévi-Strauss, 1971 e 1981) volti a spiegare le peculiarità della cultura in termini di soluzione a problemi di logica anziché in termini di soluzione a problemi d'adattamento ecologico e di funzionamento dei sistemi sociali, sono stati rari ed estemporanei. La cultura concepita come un insieme d'attività biologiche, d'azioni tecniche ed espressive, come lo specchio delle intenzioni razionali degli individui, esterna ad essi, rete di rapporti interpersonali, costruzione logica del ricercatore, messaggio, rete di comunicazione, coarcevo di rapporti di potere espressi nello scambio di merci e di servizi; la pluralità delle culture come il prodotto di permutazioni e di trasformazioni esistenti solo nella mente degli uomini, una tale concezione non ha riscosso molto successo nel mondo della ricerca.

In breve, negli ultimi lustri del secolo $\mathrm{XX}^{\circ}$ le ricerche sulla cultura non si sono discostate molto dal paradigma Tylor-Boas-Malinowski né da quello della corrente «cultura e personalità » (Sapir, Benedict, Mead) e della variante «personalità di base» (Linton, Kardiner). Grazie a queste variazioni sullo stesso paradigma si sono continuati ad approfondire i temi già elencati nel saggio di Rossi (art. CULTURA, p. 1144) nonché a descrivere le innumerevoli istituzioni e pratiche culturali, sia individuali che collettive, nei loro aspetti razionali, nelle loro differenze e relazioni, nelle loro significazioni, funzioni e conseguenze sociali. I risultati finora prodotti non hanno modificato le conoscenze correnti (Kuper 2001). Poiché gli uomini hanno la capacità d'inventare, di trasmettere e d'acquisire simboli culturali (credenze, comportamenti, patrimonio di cose e di idee), la cultura è anche strumento di comunicazione, è l'analogo del linguaggio parlato. La simbolizzazione e l'universalità del processo simbolico diventano uno degli attributi della natura umana mentre la cultura un sistema simbolico che produce simbolizzazioni specifiche e variabili da un contesto all'altro. Non esiste un'universalità delle significazioni simboliche benché la cultura sia, in un qualunque dove, la manifestazione visibile e l'espressione d'un sistema sociale organizzato. 
Le società umane esistono come insiemi discreti e le istituzioni sociali sono sistemi omeostatici. Se la cultura è un processo complesso fondato sullo scambio, sulla comunicazione, sulle interazioni, se consiste in tratti esistenti in sé, come segmentare questi tratti, distinguere i rilevanti da quelli irrilevanti? Se poi essa indica una caratteristica unica condivisa dall'umanità intera, allora la nozione è puramente ridontante e la sua definizione è tautologica. Infatti la cultura sarebbe ciò che è umano mentre l'umanità sarebbe ciò che è culturale. In più, se il comportamento è determinato dal contesto culturale, perché mai se ne valutano le conseguenze funzionali secondo criteri scientifici panumani?

Finora non è stata data una risposta a questi interrogativi. Lo scopo precipuo delle teorie diffusioniste è stato d'illustrare i contatti e gli apporti culturali indotti dalla diffusione di tecniche e d'oggetti materiali; di quelle funzionaliste, sulla base d'oggetti materiali a vocazione tecnica, essenziali per fabbricare altri oggetti, di valorizzare gli aspetti non-simbolici delle attività produttive degli uomini, di richiamare l'attenzione sulla creatività umana, sullo spazio, sul tempo, sulla socialità degli oggetti, dei materiali, delle tecniche, sulle loro funzioni nelle creazioni socio-culturali. Queste ed altre teorie hanno prodotto delle proposizioni descrittive piuttosto che esplicative, si sono fondate sul truismo che qualsiasi esperienza è mediata da un sistema simbolico (il linguaggio, la scienza, ecc.) ed hanno confuso il simbolico con l'immaginario e trasformato la cultura in un sistema proiettivo. In più, considerando l'universalismo come l'antonimo di particolarismo e l'assolutismo come quello di relativismo, hanno sottovalutato che l'apparizione differenziata d'un tipo di comportamento dipende da condizioni socio-culturali differenziate, e trascurato che le caratteristiche psicologiche panumane sono il prodotto di caratteristiche biologiche filogeneticamente determinate (l'ereditarietà) e di esigenze funzionali peculiarie a qualsiasi sistema sociale.

Le inchieste sul campo hanno mostrato che tutte le società sono complesse, anche le più piccole, che non hanno sistemi di valore comuni trasmessi mediante la socializzazione, ma anche rivelato che la cultura è una razionalizzazione prodotta da attori sociali, dotati d'una natura umana strutturata da configurazioni sensoriali, radicata nei bisogni individuali umani percepiti attraverso significazioni di simboli e sistemi simbolici di una società specifica in un'epoca particolare. Certo, i processi d'apprendimento sono complicati, dipendono dall'ambiente in cui si trovano a vivere gli individui, sono variabili; è vero che esistono molteplici sistemi di valori, alcuni propri a sottogruppi, altri a subculture locali o a gruppi particolari. Senonché i sistemi culturali, che hanno un forte grado di coerenza quando sono formati da strutture sociali elementari, nelle società complesse palesano una grande eterogeneità con caratteristiche qui compatibili là incompatibili. Appunto perciò questi sistemi vanno analizzati nei loro peculiari processi storici ed interpretati come soluzioni a problemi o risposte di attori sociali collocati in sistemi d'interazione con strutture diverse. I membri d'una società non partecipano ad una cultura comune, ad un sistema di valori comuni. Anche a supporre che gli individui interiorizzino i valori e che poi questi regolino i loro comportamenti, la cultura è una prolunga della natura, governa comportamenti quasi istintivi, talvolta sottratti allo stesso controllo del soggetto. I comportamenti non sono mai il prodotto d'un condizionamento; sono la risultante d'una intenzionalità. In conseguenza, la socializzazione non è un semplice meccanismo d'interiorizzazione, non elabora l'habitus, è piuttosto un processo d'adattamento 
a situazioni mutevoli e varie, un processo caratterizzato da decisioni e compromessi che il soggetto realizza in funzione dei bisogni da soddisfare, delle norme che gli sono imposte, dei valori e delle credenze sottoscritti, degli interessi ponderati. Il culturalismo che fa delle disposizioni la matrice dello habitus e la principale variabile esplicativa del comportamento, deve far ricorso alla petizione di principio secondo cui la cultura è la causa principale di queste stesse disposizioni (Douglas \& Ney 1998).

L'interiorizzazione completa della cultura d'un gruppo è stata detta assimilazione; l'acculturazione sarebbe la trasformazione, ed eventualmente la creazione di nuovi modelli culturali per effetto del contatto permanente con altri gruppi sociali; la deculturazione sarebbe lo sfilacciamento, il progressivo abbandono dell'originaria cultura d'appartenenza, la non ancora avvenuta assimilazione d'una nuova cultura. La fragilità di questi concetti è stata verificata. La conversione pura e semplice alla cultura dell'altro è una presupposizione gratuita giacché ogni cultura seleziona, reinterpreta, trasforma, secondo una logica propria, gli elementi presi o ricevuti, non è mai passiva di fronte ai cambiamenti culturali esogeni. L'uniformizzazione resta un giudizio ideologico e la mondializzazione culturale un mito. Solo l'etnocidio, lo sterminio volontario e programmato d'un popolo, può mettere in pericolo la sua cultura, mai eliminarla definitivamente o modificarla profondamente (Amselle 1990 e 1996).

\section{IL RINNOVAMENTO DEL CONCETTO}

Le relazioni tra il sistema sociale e la personalità a lungo considerate asimmetriche, sono da qualche anno ritenute simmetriche. Considerata come relativamente passiva, plasmata dal sistema sociale, priva d'influenza su di lui, la personalità non era che l'aspetto soggettivo della cultura. I lavori recenti riconoscono, invece, che la personalità è influenzata dal sistema sociale, ma difendono la tesi che questo è a sua volta influenzato dalla personalità, che le relazioni di scambio tra i due sono molteplici e consistenti. Dalle ricerche sulle influenze dei sistemi sociali sullo sviluppo e la formazione della personalità, gli studiosi recenti deducono le modalità mediante cui le personalità modificano il sistema sociale, secondo quali processi, nel corso della socializzazione, creano bisogni comuni e personalità modali, basi psicologiche equipollenti alle motivazioni culturali. Se i bisogni sono soddisfatti mercé ruoli assunti conformemente alle norme culturali, il funzionamento della società è assicurato. In questa prospettiva la personalità è lo strumento capitale per garantire il controllo sociale. Le sanzioni sono efficaci se diventano bisogni che motivano l'esecuzione del ruolo. Le norme culturali che la prescrivono devono essere interiorizzate affinché la non-conformità provochi ansietà e la conformità alla norma diventi bisogno motivante l'esecuzione del ruolo. I fini prescritti raggiunti coll'esecuzione d'un ruolo sono poi investiti e servono ai bisogni personali per motivare le ulteriori esecuzioni di ruoli numerosi e complessi.

Abbandonato il paradigma secondo cui la cultura è il determinante indifferenziato ed esclusivo della personalità e dei comportamenti grazie al processo d'interiorizzazione (Spiro 1987), gli studi recenti considerano che la personalità non è consustanziale alla cultura né isomorfa ai comportamenti. Sistema di disposizioni 
cognitive, affettive, percettive e motivazionali, la cultura include pattern di comportamenti e tratti di personalità. La sua variabilità è associata alla variabilità della personalità. L'uomo è un organismo biologico ma è anche un animale psicologico e sociale, produttore di processi coscienti ed inconscienti. La conoscenza di questi processi permette di comprendere i significati apparenti, le funzioni manifeste dei simboli culturali come le loro significazioni profonde e funzioni latenti.

Questi tentativi di rifondare la nozione di cultura sono stati più o meno ispirati dall'approccio praticato dalle scienze cognitive per le quali, com'è noto, sia i sistemi naturali che quelli artificiali, hanno in comune il percepire, il comprendere, il risolvere i problemi dell'azione. Secondo il punto di vista cognitivo sono importanti la struttura dell'informazione e le funzioni che permettono gli atti di conoscenza. Perciò gli studiosi di questa tendenza riservano un'attenzione particolare a processi mentali differenti (la percezione, la memoria, il linguaggio, il ragionamento, la coscienza, le emozioni, le rappresentazioni sociali) ed alle strategie per la risoluzione dei problemi effettuate da moduli cognitivi specializzati, interagenti e cooperanti fra di loro (trattamento delle informazioni, in parallelo o in serie, cooperazione tra strutture biologiche e funzioni cognitive, pilotaggio centrale e autoregolazione).

Sin dagli anni '80, l'approccio connessionista di questo modello cognitivista ha analizzato le elaborazioni mentali in termini di reti neuronali, ha messo in evidenza il fatto che attraverso la percezione ricomponiamo ed organizziamo i dati del mondo esterno, che la memoria filtra e ricompone il passato, che non procede per semplice accumulazione, che la ragione umana non è identica alla logica, che essa utilizza delle procedure euristiche ( dei miniprogrammi per la risoluzione dei problemi ) e degli schemi mentali ( delle rappresentazioni stabili che permettono di decodificare il reale ). Il mondo è quello che i nostri sensi permettono di «vedere», che le basi innate del linguaggio esprimono. La visione della società è modellata dalle rappresentazioni sociali che ci rendono sensibili a certi aspetti dell'ambiente e che sono strutturate secondo noduli stabili. Si sa che in tutti gli esseri umani l'emisfero sinistro del cervello presiede alle funzioni analitiche (linguaggio, ragionamento, calcolo), mentre l'emisfero destro regola le funzioni analogiche (mappe mentali dell'ambiente circostante, i giudizi estetici, la musicalità) nonché le manifestazioni esterne delle emozioni (piacere, dolore, rabbia, invidia, gelosia, ecc.).

Col superamento dell'opposizione tra la descrizione normativa e razionale del pensiero e la descrizione psicologica del pensare, il modello cognitivista offre una nuova teoria della natura umana. Dato che esiste un livello intermedio di rappresentazione o conoscenza tra il mondo fisico e cerebrale e quello comportamentale, non è più possibile derivare dai concetti mentali, posti all'origine, le proprietà dei loro contenuti, per poi caratterizzarli in termini puramente descrittivi e causali. Si riconosce così che le nostre capacità intellettuali sono basate parimenti su conoscenze tacite standard e su processi sub-personali o sub-individuali. Finora però non è stato possibile naturalizzare queste capacità giacché non si è in grado di comprendere e spiegare se e in che maniera delle catene causali colleghino i contenuti del pensiero. Trattasi d'un problema importante ma difficile dato che il pensiero è fatto da una grande quantità di sottomeccanismi specializzati, da moduli constitutivi dell'equipaggiamento genetico stesso. Invero, in tutti i contesti culturali si acquisisce, in maniera spontanea e ad un tempo particolare e simile, 
il concetto di specie biologica. Laddove uno o più moduli sono deteriorati o assenti, si constata un deficit cognitivo localizzato. L'autismo è prodotto da un deficit della capacità d'attribuire ad altri stati mentali mentre talune lesioni cerebrali producono l'incapacità di denominare le piante o riconoscere $\mathrm{i}$ visi.

I fenomeni sociali hanno delle componenti diverse ma esiste un legame causale che caratterizza il cervello e lo spirito, marcati ambedue dall'ambiente e quest'ultimo a sua volta marcato dall'attività d'altri umani. Queste concatenazioni tra i processi interni del cervello ed i processi esterni al livello delle popolazioni, restano mal conosciuti, ancora tutti da elucidare.

La variabilità culturale dei comportamenti e delle personalità non prova che l'organismo (genotipo e fenotipo) è una scatola vuota, che non esistono caratteristiche invarianti pan-umane. Al di là delle specificità e delle differenze ambientali e storiche, tutti gli uomini, in qualsiasi società, hanno tratti biologici comuni ed analoghi processi d'adattamento. Le caratteristiche biologiche, sociali, culturali alla base del processo di socializzazione (il controllo delle attività corporali e delle frustrazioni, la distinzione tra l'immaginario ed il reale, ecc.), sono dappertutto le stesse e formano un insieme di costanti che in interazioni reciproche compongono la natura umana universale.

Le ricerche in corso stanno, dunque, abbandonando la tesi dell'isomorfismo tra la cultura, il comportamento e la personalità, alla base del modello dell'interiorizzazione della cultura, e indagano invece i rapporti complessi esistenti tra il genotipo ed il fenotipo, tra la natura e la cultura, tra la personalità ed i comportamenti, tra l'individuo e la sua società.

\section{DALLA CULTURA ALLE CULTURE}

Poiché la cultura esiste grazie agli individui in situazioni d'interazioni durabili, poiché ogni contesto impone le sue proprie convenzioni ed un sistema d'attese, le ricerche sui processi interattivi che producono i sistemi culturali hanno avuto, in questi ultimi anni, un notevole sviluppo. Esse hanno rivelato l'eterogeneità e l'instabilità di tutte le culture e messo in evidenza le logiche dei comportamenti apparentemente contraddittori degli individui agenti in contesti diversi. Il tema tradizionale della gerarchia delle culture, della cultura dominante o egemonica e delle culture dominate o subalterne, le teorie oliste delle culture nazionali, regionali e micro-locali, hanno subito trasformazioni così radicali che ormai molti ricercatori li giudicano alla stregua d'una semplice metafora.

Un sistema culturale non può essere né superiore né inferiore ad un altro. Tutti i sistemi hanno una propria peculiarità, non esistono gerarchie culturali. Il che non vuol dire che tutti i gruppi sociali sono eguali e tutte le culture equivalenti. Sono le ineguaglianze economico-sociali e politiche, i rapporti di forza a creare le gerarchie sociali, le quali poi tentano di valorizzare, in maniera più o meno arbitraria, un dato ordine culturale e d'imporlo come egemonico. I subalterni dispongono di numerose possibilità sia per resistere sia per opporsi, possono reinterpretare le produzioni culturali imposte e cosi facendo trasformarne i contenuti. I rapporti simbolici non funzionano secondo la stessa logica dei rapporti sociali. L'egemonia culturale è basata sul consenso, quindi non è né costante, né durevole, mai permanente. Deve essere costantemente ottenuta attraverso un'opera d'incul- 
cazione, di persuasione, di convinzione. I risultanti non sono né scontati né univoci, possono persino essere perversi rispetto alle attese dei dominanti. I rapporti di dominazione culturale non si confondono quasi mai coi rapporti di dominazione sociale perché funzionano secondo una logica ed una coerenza propria, perché arrivano a conservare una certa autonomia relativa.

Le ricerche sulla cultura materiale, strettamente legata colla vita economica ma da essa distinta, lo studio degli oggetti, degli utensili, dei manufatti, ha fatto luce sui meccanismi dell'esistenza quotidiana, quella che assorbe gli atti ed i pensieri degli esseri umani, quella che è indissociabile dal lavoro e dalla produzione, ed ha inoltre circonscritto il terreno su cui opera l'economia, la materia che essa lavora, i livelli tecnici che ne costituiscono la base. Queste ricerche hanno avuto delle incidenze maggiori anche sugli studi delle culture popolari.

Di quest'ultime sono state esplicitate le logiche operative, le combinazioni d'operazioni con cui quelle elaborano le maniere di pensare, di rendere vivibili le condizioni oggettive di vita dei propri membri. Tutte le azioni sociali sono sempre datate e territorializzate, è la localizzazione delle azioni umane nello spazio sociale e nel tempo storico a configurare nel comportamento sociale tutto ciò che può essere compreso come un'azione peculiare (Grignon \& Passeron 1989; de Certeau 1980 e 1993). La descrizione della democratizzazione culturale prodotta dalla scuola, l'analisi delle incidenze dei prodotti delle industrie culturali, della mondializzazione, della perdita di legittimità della cultura intellettuale classica, hanno rivelato la fallacia del romanticismo populista, della visione essenzialista delle classi popolari (Lasch 1981), ed hanno dimostrato inoltre che la cultura popolare, quella della gente ordinaria, non è mai passiva, dispone d'una ampia autonomia nell'interpretazione e nell'accettazione, nella riappropriazione e nella trasformazione dei beni culturali prodotti e diffusi dal processo industriale di produzione o dalla classe egemonica. Le identità collettive popolari non sono assorbite o manipolate dai media, la cultura popolare non si fonde e non si confonde colla cultura di massa. I prodotti uniformi di questa cultura sono rielaborati, re-interpretati sino a dotarli d'altri significati, a renderli incontrollabili e resistenti (Couldrey 2000; Storey 2001).

Le ricerche di Richard Hoggart (1957) hanno analizzato la cultura popolare come una strategia di resistenza agli effetti congiunti della cultura dominante e della cultura di massa. La descrizione degli oggetti culturali elaborati da quella cultura, delle «maniere di fare e d'essere» prodotti dalle esperienze della vita sociale, dell'adesione ai valori collettivi, del rifiuto di quelli individualisti, della coerenza delle pratiche e dei comportamenti, hanno aperto la via alle ricerche, prima coordinate dal «Center for Contemporary Cultural Studies», creato a Birmingham nel 1963 da Richard Hoggart, e poi sviluppatesi negli Stati Uniti secondo altre prospettive ed interessi, in difesa d'ogni specie di minoranze. Quest'ultimi lavori sono all'origine di forme nuove di radicalismo, del rifiuto delle gerarchie e dell'elitismo, della scienza univerale, di difese, talora apologetiche, dei gruppi sociali minoritari, marginali. Le «Cultural Studies » denunciano la dissoluzione della cultura popolare nella cultura di massa, la massificazione di tutte le pratiche culturali, l'ideologia scientista e sessista, l'influenza preminente dei mass-media sulle abitudini, sui valori morali, sui processi di socializzazione (Willis, 1990). Mentre Hoggart ed i suoi primi discepoli ritenevano che esiste una maniera popolare d'assorbire, anche intensamente, i prodotti dell'industria cultu- 
rale, che le capacità di resistenza ai cambiamenti, all'industrializzazione degli oggetti simbolici e culturali, d'imporli al consumo di massa, sono considerevoli, gli studiosi americani, più radicali ed estremisti, partono dall'idea che viviamo una contemporaneità senza precedenti, la quale anticipa un futuro radicalmente diverso, fatto di dominazione e di disumanizzazione. Attribuiscono legittimità e validità a tutte le esperienze, a tutte le pratiche sociali, ritenute eguali, s'oppongono alla divisione sessuale e razziale, e mirano a dare il potere («to Empower», «the Empowerment») ai gruppi sociali finora discriminati, ai giovani, alle donne, agli omosessuali, ai neri, agli ispanici, agli indios, ecc.( McRobbie 1991) ed agli individui che esplorano nuove possibilità di vita e maniere d'essere e di fare innovative. I movimenti anti-culturali hanno considerato i comportamenti di rigetto e di contestazione dei modelli vigenti manifestazioni e avanguardia annunciatrice d'una nuova cultura. Theodore Roszak (1969) ne ha fatto la teoria e ne ha descritto i tratti più tipici: lo stile di pensiero mistico ed olista che si oppone all'orientamento strettamente razionalista ed atomista del pensiero scientifico; l'adozione d'un modo di vita anticonformista, edonista, antimaterialista, la ricerca d'una società alternativa anti-autoritaria, in armonia colla natura, liberata dall'etica del consumo e del lavoro; rigetto dell'azione politica organizzata ritenuta una forma di collaborazione colla cultura dominante. Da qualche anno però alcuni studiosi sono più riservati e riconoscono che gli «hooligans », gli « urban Riots », i « sauvageons », non sono eroi ribelli o resistenti alle regole sociali ma piuttosto tirannelli oppressori e talvolta anche delinquenti (Campbell 1995).

Anche gli studi sulla cultura di massa sono stati profondamente rimescolati da questi rinnovamenti concettuali. Le teorie di T. W. Adorno e di H, Marcuse, che hanno confuso la cultura per le masse colla cultura delle masse, le loro dottrine sull'alienazione culturale, sulla sterilizzazione delle capacità creative degli individui, sull'impossibilità di sottrarsi alla dittatura dei mass-media, sul livellamento e sull'uniformizzazione operati dai mezzi di comunicazione di massa, si sono rivelate inconsistenti, per non dire fallaci. È vero che i messaggi mediatici sono standardizzati, uniformizzati, ma è altrettanto vero che essi non sono ricevuti in maniera analoga dai pubblici cui si rivolgono. Le inchieste sociologiche, analizzando i discorsi e le immagini diffuse, hanno rivelato che la ricezione è diversa da un pubblico all'altro, da un contesto ad un altro. Qui producono polemica o indignazione, là attenzione scettica o accettazione distratta, altrove atteggiamenti indifferenti, riletture pregne di riserve mentali, comprensioni parodistiche, sarcastiche o semplicemente indifferenti o ironizzanti. Per queste ragioni, adesso, abbandonate le grandi costruzioni teoriche, si studia ciò che i consumatori fanno di ciò che consumano e si comincia a capire perché la globalizzazione massmediatica non produce la mondializzazione intesa come l'omogeneizzazione di tutte le culture. Al contrario, lo stesso messaggio, ricevuto in diversi contesti, visto in determinate situazioni, produce effetti diversi, genera differenze sostanziali, reazioni contrastanti.

Tutti questi cambiamenti concettuali hanno ristretto la portata della vecchia nozione di cultura di classe, meglio, le hanno dato nuove prospettive. Abbandonati i contenuti filosofici ed ideologici di cui la tradizione marxista l'aveva dotata, la nozione rimanda oggi, innanzitutto, ai sistemi di valori, ai modelli di comportamento, alle pratiche quotidiane ordinarie (consumi, stili d'alimentazione, tecniche corporali, ecc.) che si osservano in gruppi sociali aventi lo stesso livello di reddito, 
le stesse abitudini di spesa, la stessa concezione del mondo, la stessa adesione a sistemi di valore, lo stesso senso d'appartenenza ad una comunità di vita e di destino, la stessa «privatizzazione» dei modi di vita.

Analoghe trasformazioni concettuali si sono avverate negli usi della nozione di cultura politica. Essa ormai è alla base di ricerche che descrivono le opinioni relativamente stabili dei diversi gruppi sociali, $\mathrm{i}$ loro atteggiamenti nei riguardi del controllo sociale, nonché i loro modelli normativi, i valori che guidano le loro scelte politiche, la concezione che hanno della legittimità e degli stili di vita della classe politica di governo e d'opposizione.

\section{IDENTITÀ, ALTERITÀ, EQUIVALENZE CULTURALI}

Sin dagli anni `60 il tema delle identità culturali s’è sviluppato senza tenere conto delle acquisizioni delle scienze sociali. I ricercatori di quel settore hanno tentato soprattutto di chiarire i problemi sollevati dall'integrazione sociale degli immigranti, d'accertare le modifiche o gli sconvolgimenti apportati da essi alla composizione culturale della società civile, al mercato del lavoro, alla convivenza comunitaria, di valutare i risultati delle procedure d'assimilazione, di descrivere, infine, le eventuali gerarchie socio-culturali in via di costituzione (Poutignet \& Streiff-Fenart 1995).

L'ipotesi allora dominante tra i ricercatori, era che l'identità culturale, stabile ed immutevole, determinerebbe le condotte individuali. Essa non dipenderebbe dal contesto delle interelazioni poiché sarebbe il nocciolo duro dell'identità sociale stessa. In più dell'attribuzione dell'identità gruppale, della categorizzazione della distinzione noi/essi, dell'elaborazione di diverse differenziazioni contrapposte a quelle degli altri, l'identità culturale fisserebbe, quindi, l'appartenenza primordiale e definitiva dell'individuo ad un determinato sistema sociale. Gli individui non sarebbero, da soli, capaci di autodefinirsi né di costruire la loro propria identità sulla base di criteri scelti e combinati poi liberamente in funzione delle soluzioni da dare ai problemi posti dalle interelazioni e dai contesti in cui si deve agire.

In verità, tutte queste ricerche sono state influenzate dalla logica dello StatoNazione e dal presupposto che un gruppo sociale o una comunità seleziona, in maniera sovrana, le sue tradizioni, interpreta arbitrariamente la sua storia ed il suo presente, fissa i modelli normativi dei comportamenti, fattori indispensabili per realizzare l'ordine sociale e per tenere unita la società. Lo Stato ne sarebbe l'artefice ed il garante mediante l'unione della cittadinanza colla nazionalità, grazie alla regolamentazione ed al controllo di questa identità, divenuta riferimento esclusivo, assunta come la sola legittima. Ed è così che un'identità culturale può essere percepita come positiva mentre un'altra negativa; ed è così che si creano le premesse per escludere le identità culturali differenti, per creare, in situazioni estreme, le condizioni che sboccano poi nelle purificazioni etniche.

L'insieme di queste ricerche può essere raggruppato, molto sommariamente, in tre tendenze maggiori. La prima dà un contenuto oggettivo all'identità culturale, la fonda geneticamente e naturalizza l'appartenenza culturale. L'identità è data dal gruppo d'appartenenza, che pre-esiste all'individuo, che la riceve più o meno passivamente, che deve sottomettervisi, pena la marginalità o lo sradica- 
mento. Una tale identità non può evolvere perché è innata, è iscritta nel patrimonio genetico. Il sentimento d'appartenenza è reputato innato e l'identità culturale diventa un prerequisito immanente che caratterizza e definisce, in maniera stabile e definitiva, l'individuo. Questa eredità culturale è trasmessa, consolidata e allargata dal processo di socializzazione nel corso del quale i modelli culturali sono interiorizzati. L'identità è, per conseguenza, consustanziale ad una cultura particolare. Qui le emozioni e le solidarietà condivise strutturano l'identificazione al gruppo e contribuiscono a complessificare i meccanismi d'identificazione collettivi ed individuali. I ricercatori di questa tendenza sono convinti che l'appartenenza ad un gruppo etnico determina e condiziona l'appartenenza sociale. Perciò l'identità, configurata sulla base di criteri determinanti, oggettivi (l'eredità, le genealogia, la lingua, la personalità di base, il rapporto con un dato territorio, ecc.), è necessariamente una proprietà del gruppo, è trasmessa indipendentemente da quelle degli altri gruppi etno-culturali e sociali. Un gruppo sprovvisto d'una lingua propria, senza un territorio, senza un fenotipo culturale, non costituisce una comunità etno-culturale, non può rivendicare un'identità culturale peculiare, autentica. La seconda tendenza ha una visione più soggettivistica dell'identità. Questa non è data una volta per tutte, proviene piuttosto dal sentimento d'appartenere ad una collettività alla quale ci si identifica. Le rappresentazioni che gli individui si fanno della realtà sociale e delle sue divisioni contribuiscono a dare delle caratteristiche alle identità culturali. Perciò esse sono variabili, effimere, dipendenti da scelte individuali operate in contesti relazionali e situazionali momentanei e peculiari. Studiate dalla psicologia, dall'antropologia, dalla sociologia e dalle scienze storiche, le identità culturali non sono altro che delle costruzioni - rappresentazioni di cui gli individui si servono per dare un senso al loro essere al mondo. La terza tendenza postula che l'identità si costruisce e si ricostruisce a seconda delle situazioni, mediante un susseguirsi di scambi sociali. Non esiste un'identità in sé, trattasi d'un rapporto con altri che si forma mediante un sistema di relazioni e di strategie svolgentisi in contesti e situazioni specifici. Gli individui partecipano a diverse culture, fabbricano, con diversi materiali prodotti in situazioni specifiche, le loro identità personali, e le argomentano sincreticamente. In altri termini, l'identità è costruita e come tutte le costruzioni sociali prende la forma d'una rappresentazione. Riflette la complessità sociale ed i rapporti di forza del momento, le lotte per produrli o riprodurli o per rovesciarli. È multidimensionale, dinamica, difficile da circonscrivere e da definire. Mezzo per raggiungere un fine, essa è mutevole e relativa, si presta a molteplici interpretazioni ed anche ad innumerevoli manipolazioni. La partecipazione ad una cultura non implica automaticamente il possedere un'identità particolare giacché non esiste un'identità in sé e per sé. Persino l'identità etno-culturale utilizza alcuni elementi d'una cultura, in nessun caso l'insieme delle componenti d'una stessa cultura. Quest'ultima può persino essere strumentalizzata differentemente ed a seconda delle diverse strategie d'identificazione. Donde la necessità di stabilire, di volta in volta, cosa significhi, in un dato momento, in un certo contesto, mantenere, difendere, proteggere o mettere in questione un'identità particolare. Questa presa in conto del pluralismo delle identità, dei sistemi di valori soggiacenti, della partecipazione a diverse culture, ridefinisce l'identità universale in termini d'identità differenziata e proclama fallace il dibattito sul multiculturalismo relativista e sull'universalismo dell'esclusione. Il che conduce altresì a riconoscere 
l'uguale dignità di tutte le identità culturali, pur nella differenza e nella diversità, ad attribuire all'alterità valore e legittimità. La nozione di «cultura societale» (Kymlicka 1995) sottolinea che tutte le maniere di vivere sono significative se prodotte dalla storia, dai sistemi rappresentativi, dai saperi e dalle saggezze tacite nelle sfere d'attività comunitarie, per cui sono tutte meritevoli di garanzie che ne assicurino la riproduzione e ne favoriscano l'esercizio.

Queste ricerche sull'identità culturale hanno suscitato interrogazioni, perplessità e alimentato un dibattito che è lontano dall'estinguersi. Se la teoria dell'esistenza d'identità culturali naturalizzate, oggettivate, non suscita né nuove questioni né risposte convincenti e concordanti collo stato delle conoscenze attuali, la corrente soggettivistica e quella dell'identità multipla sono, invece, all'origine d'un dibattito di più in più animato quasi esclusivamente dai filosofi sociali e dagli scienziati della politica. Wilson (1985), per esempio, è dell'avviso che la mondializzazione e la globalizzazione privano gli individui dei legami comunitari, sostituiti da rapporti sociali multipli e funzionali, insufficienti ed inadatti a mobilitare gli individui, a riconoscere i valori ultimi. Ma se esistono tanti valori quante sono le comunità, allora i valori culturali hanno un solo fondamento, quello di essere il cemento comunitario. Dal canto suo Beck (1993) sostiene che l'individuo incontra oggi innumerevoli ostacoli nella costruzione del self dato che tutte le sue decisioni sono prese sotto la pressione dell'ambiente. Sottomesso a costrizioni strutturali, la sua autonomia è ormai illusoria, le sue decisioni sono reazioni e non già delle scelte razionali. La mondializzazione ha prodotto del nihilismo. Il soggetto sociale non è più il depositario e la fonte dei valori, è niente altro che un semplice meccanismo delle strutture sociali. Giddens (1999), a sua volta, aggiunge che gli effetti strutturali della mondializzazione hanno prodotto una società in rottura con le società del passato, caratterizzata da una discontinuità radicale rispetto alla società industriale moderna. I criteri che permettevano d'attribuire valore positivo o negativo alle cose, che davano un contenuto all'identità culturale, sono adesso divenuti inconsistenti. Se il legame collettivo nasce quando gli individui fanno prova di virtù, di moderazione, di tolleranza, d'ossequio alle leggi, di rispetto dell'autonomia degli altri, insomma di ciò che Rawls (1993) chiama raisonabless, vale a dire il senso del ragionevole e del giusto, allora come conciliare i diritti culturali particolaristici con quelli generali, i diritti individuali con quelli collettivi, evitare la formazione di comunità chiuse su se stesse ed anche che talune minoranze pratichino l'esclusione al fine di preservare la propria integrità culturale?

Gli studiosi post-modernisti ritengono questa problematica non pertinente. La relativizzazione dei messaggi culturali nazionali, i modi di vita imposti dalle logiche mercantili, il rafforzamento dell'individualismo, la variazione dei modi di vita e l'ideologia dell'interiorità e della comunicazione, hanno reso caduca e dimostrato la fallacia della definizione unitaria del vivere insieme, della società unificata, d'identità culturali stabili. Le società post-moderne producono una reificazione definitiva delle identità, una codificazione di forme particolari di pluralità culturali, una forma illusoria d'unità sociale, d'equivalenza delle culture, colle quali si crede mascherare la tirannia d'identità molteplici, sfuggenti, inafferrabili ed inconsistenti.

Se la cultura è l'apprendistato delle libertà individuali e la democrazia quello della volontà generale, se la cultura è una configurazione dell'essere e la 
democrazia un'organizzazione dell'esistenza, se la prima è l'equilibrio invisibile delle cose che ci aiutano a vivere e la seconda è l'ordine visibile di quelle che ci governano e ci fanno interagire convenientemente, cosa bisogna intendere per culture equivalenti e per identità culturali multidimensionali e differenziate ? Se l'individuo ha la capacità ed il diritto di giudicare, d'accettare e di rifiutare i valori, i progetti e le norme del suo ambiente sociale e culturale; se l'individuo è l'artefice d'identità e può creare all'infinito delle diversità e delle differenze, non si rischia d'accordare lo stesso riconoscimento a qualsiasi rappresentazione quali che siano i comportamenti ed i valori praticati? Se la società è un insieme organico, la cui coesione è garantita dall'esistenza di similitudini tra i suoi membri, dalla condivisione di valori comuni, da una stessa eredità storica, da un analogo conformismo dei comportamenti, se le differenze innumerevoli provocano pericolose frammentazioni sociali ed un relativismo culturale caotico, accettare qualsiasi differenza, ascrittiva/ereditaria e acquisita, nella sfera pubblica ed in quella privata, trattare gli individui portatori di valori, costruttori d'identità specifiche, implica che bisogna ignorare e negligere il fatto che esistono valori ineguali ma anche che le differenze espresse liberamente nella sfera pubblica possono ledere la libertà degli altri. Se non esiste una simmetria tra identità pubbliche ed identità private, tra cultura a vocazione universale e cultura a vocazione particolaristica, in che modo allora garantire le specificità, le particolarità, le alterità, le pluralità culturali e le identità collettive ed individuali in una società democratica a vocazione universalistica, vivente ed agente perché le sue fondamenta poggiano su un principio d'unità e d'identità, di cui purtroppo è sprovvista la democrazia procedurale ed anche il patriottismo costituzionale strutturato dall'adesione volontaria a valori astratti e formalizzati.

\section{DIFFERENZIALISMO E PLURALISMO CULTURALI IN UNA SOCIETÀ DEMOCRATICA}

Posto anche che la cultura non si trasmette geneticamente, che è una costruzione sociale in stretta correlazione col contesto sociale in cui si vive e si agisce, ne consegue che essa è fatta di faccette multiple e varie, di pari o di analoga dignità. In questo caso tutte le identità culturali hanno diritto alla medesima eguaglianza di trattamento e tutte le culture sono equivalenti. Le loro rivendicazioni dell'uguaglianza economico-sociale, del riconoscimento delle differenze dei loro sistemi simbolici, non possono allora non trovare uno sbocco nel sistema politico.

In che maniera conciliare l'uguaglianza universale di tutti i cittadini con il riconoscimento d'identità particolari, con le rivendicazioni particolaristiche di queste identità?

Le risposte ad una tale questioni sono state finora diverse. I liberali sostengono che esiste una sfera (società civile, vita privata) dove si esprimono liberamente orientamenti culturali differenti. Là si è liberi d'accettare o di rifiutare $\mathrm{i}$ valori, $\mathrm{i}$ progetti e le norme degli altri, di valorizzare e praticare i propri ritenuti più pertinenti e validi. Nella sfera pubblica, invece, l'appartenenza, la cittadinanza, l'uguaglianza, l'autonomia, la sicurezza, la protezione delle libertà fondamentali restano preponderanti; qui è preminente la necessità ed il diritto di regolamentare tutte le differenze onde prevenire o impedire che esse ledano le libertà delle altre 
identità culturali (Holmes 1993). I repubblicani constatano che il potere è confiscato da politici di professione e da burocrati irresponsabili, che i cittadini sono passivi, indifferenti alla vita pubblica, egoisti, disobbedienti, con desideri e bisogni illimitati. Se la cittadinanza è partecipazione, è amore della collettività e del bene comune, rifiuto della divisione del corpo sociale, allora bisogna formare dei cittadini, inculcare in essi l'amore della collettività e dell'uguaglianza, il senso dei limiti. Credere che tutti possano accedere autonomamente al sapere ed alla razionalità, che la sfera privata e la sfera pubblica esistano indipendentemente dai rapporti di forza, è niente altro che la maniera retorica di celebrare «the Santification of ordinary Life» (Taylor 1989). Le interazioni sociali producono ineguaglianze (razzismo, sessismo, statuti inferiori, gerarchie d'ogni tipo, discriminazioni linguistiche e d'origine sociale, limitate opportunità sul mercato del lavoro e della politica, ecc.). Se lo Stato si astiene dall'intervenire, resta neutro nel funzionamento del sistema culturale (scuola, mass-media, memorie e narrazioni storiche), queste ineguaglianze diventano sempre più consistenti. Al contrario, deve essere il promotore di programmi di riparazione, di «Affermative action», di «Recognition »; deve favorire le espressioni delle orientazioni culturali di tutte le minoranze, riconoscere che le identità culturali minoritarie, anche quelle più colpite dai pregiudizi sociali, hanno una pari dignità, costitutiva dell'essere umano, nell'appartenenza alla società.

Molti studiosi considerano la neutralità culturale dello Stato un'ideologia elaborata per confortare le maggioranze culturali, il «Charter Group». La cosiddetta «cultura nazionale», che lo Stato dichiara essere il prodotto d'una esperienza storica comune, d'una condivisione permanente della stessa genealogia, è ritenuta una interpretazione della storia e del presente, una selezione arbitraria delle tradizioni, una imposizione. Dal canto loro, i comunitari (Mac Intyre 1981) rigettano gli approcci liberali e repubblicani perché sarebbero costruiti su una concezione irrealistica, astorica, quella che fà dell'individuo un essere razionale, capace di scelte ragionevoli, di strategie deliberate, di fabbricare la sua identità culturale ed il suo essere al mondo, come se non fossero la risultante di esperienze sociali collettive a lui anteriori. Il radicamento sociale e culturale in una comunità è costitutivo dell'essere umano. Il soggetto sociale non è autonomo, non esiste prima della collettività cui appartiene, le sue scelte sono condizionate da regole indipendenti dalla sua volontà. La sua identità si forma mediante interazioni individuali e collettive, nel presente e col passato, e non ha esistenza se gli altri non la riconoscono e non la confermano. È la comunità in cui è nato, in cui vive, è questa a procurargli valori e riferimenti, il senso d'appartenenza ad una collettività, le possibilità d'attribuire e distribuire significazioni, darsi delle finalità, realizzare progetti con una certa autonomia.

Tutti questi studi sulle identità culturali hanno aperto un nuovo campo di ricerche sulla giustizia sociale, sui diritti alla protezione delle culture delle minoranze etniche, sessuali, dei Neri, degli Ispanici, degli immigrati e delle donne; sono all'origine d'un vivacissimo dibattito sulla «Redistributive justice versus Reparative justice», sui programmi d'azione positiva in favore delle persone che subiscono forme d'esclusione e di marginalizzazione.

Per alcuni, introdurre un trattamento speciale, cioè il riconoscimento e la protezione pubblici delle differenze degli orientamenti culturali individuali e collettivi, significa storcere, se non distruggere il principio d'uguaglianza, intro- 
durre una discriminazione pericolosa e lesiva della coesione sociale. In più, nessuno può definire, in maniera generale, accettabile da tutti, ciò che è la vita buona e ciò che è il bene essenziale da rispettare per vivere in società. I criteri per definirli sono innumerevoli, variabili e negoziabili di caso in caso, di volta in volta, a seconda dei contesti e delle circostanze, mediante regole di funzionamento accettate consensualmente (Gray 2001). La tolleranza ed il rispetto, la libertà d'espressione delle minoranze sono, certo, elementi costitutivi d'una società libera e democratica. Tuttavia una politica stabile orientata a proteggere e sostenere le minoranze non consoliderebbe né estenderebbe quegli elementi. Le discriminazioni positive non creano diritti culturali positivi ma accrescono le differenze tra la minoranza, tra le altre minoranze e con la maggioranza.

Per altri, il riconoscimento del pluralismo culturale non dovrebbe porre problemi particolari alle società liberal-democratiche. Bisogna tuttavia essere coscienti che la genesi recente di molte identità culturali non si trova nel bisogno d'identificazione ad una cultura visssuta bensì in motivazioni particolaristiche manifestantesi allorché i valori universalistici mettono in difficoltà gli interessi d'un gruppo. Per queste ragioni, la cittadinanza, deve essere il principio fondante e preponderante della democrazia, più importante e rilevante dell'identità culturale. Una società vive, le sue istituzioni sono legittime, se esistono interessi e scelte collettive, se la partecipazione alla vita politica e sociale è vivace, se gli individui s'identificano ad esse e se ne sentono responsabili. Ė stato sovente ripetuto (Tylor 1989, 1991, 1992, 1994, 1996, 1997) che il «Self» e la «Modern Identity » s'affermano allorché l'accettazione e la pratica della cultura della società in cui si vive non sono minacciate dal rifiuto o dall'irrispetto della dignità di taluni. Da un altro versante (Walzer 1980, 1983) è proclamato che la libertà astratta non garantisce la vita in comune. Questa è possibile solo a condizione che i valori sociali, culturali, razziali, le identità specifiche siano riconosciuti ed abbiano pari dignità e diritti. La giustizia è realizzabile a condizione che si rispettino e si prendano in considerazione positiva i diversi principi che una cultura impiega per dar senso a settori della vita socio-culturale quali, ad esempio, la scuola, il mercato del lavoro, la solidarietà, l'onore, la lealtà, la gratitudine, ecc. Poiché non possiamo in nessun modo gerarchizzare gli universi culturali e dato che le sfere d'azione sono molteplici, la giustizia e l'equità obbligano a tenerle tutte in conto, ad aiutare con programmi ad hoc le identità culturali particolari, persino quelle etniche, a rispettare tutti i diritti ed i significati specifici, le valutazioni conferiti e valorizzati dalle minoranze culturali. Walzer (1995) sostiene che le autonomie locali, il federalismo e diverse forme di consociazionismo, sostenuti da una legislazione antidiscriminatoria, da intense procedure democratiche, dall'educazione interculturale, bastano a garantire la vita, il rispetto e la riproduzione delle culture minoritarie. I più scettici ritengono che laddove i meccanismi d'integrazione sociale sono troppo elastici, i rischi d'etnificazione, di razzismo, di comportamenti illiberali, di odii culturali, di chiusure etniche, sono inevitabili. Ammettere che tutte le identità culturali sono equivalenti implica la frammentazione del tessuto sociale, l'isolamento degli individui, divisioni irrimediabili. Anziché valorizzare ciò che differenzia le diverse componenti della società, è più saggio ed opportuno celebrare ciò che le avvicina, le integra e le può tenere unite. Il legame sociale riposa su regole che ci diamo, che reggono i nostri rapporti, che ci permettono di gestire situazioni conflittuali e rendere possibile la cooperazione quando gli attori hanno interessi 
divergenti, od anche antagonistici. Per ottenere un sistema d'attese tra persone con comportamenti differenti, bisogna disporre di procedure che permettano la coordinazione delle divergenze, disporre di regole che diventino delle abitudini, del «common Knowledge», delle culture, ed è ciò precisamente che fonda il sociale. Gli accordi tra le persone sono i prodotti di queste regole, di mediazioni e di convenzioni. Soltanto la politica in quanto razionalità del valore dei fini, in quanto trascendenza d'identità particolari, d'interessi settoriali, può sovvenire a tali necessità, al vivere nell'incertezza, a garantire strutture di vita ragionevoli ed adattabili, a tutti modalità d'accesso alle risorse ed all'informazione, possibilità per ognuno di re-investire le esperienze sociali ed i progetti in un rapporto proficuo al mondo ed alle cose. Le ricerche sulla cultura, sulla pluralità delle culture, sulle identità personali e collettive sono, nel corso degli ultimi lustri, sboccate nel campo della filosofia politica e sono all'origine di rigogliosi dibattiti sull'universalismo, sull'uguaglianza, sul rapporto cittadinanza-nazionalità, sull'estensione dei diritti alle persone in quanto tali. Le prospettive intravedibili non sono però nette. Nondimeno un'idea sembra intravedersi, distaccarsi con più nettezza, ed è quella che soltanto la democrazia e la cittadinanza costituzionale sono i fondamenti solidi, legittimi, d'identificazioni non esclusive, limitative e controproduttive (Schnapper 1994, 1998).

Riconoscere le differenze è conciliabile col principio di non discriminazione, che impone di non prendere in conto le differenze generiche, etniche, culturali, di trattare tutti gli esseri umani come eguali, in maniera identica? Negare le differenze conduce a trattare in modo omogeneo individui eterogenei. Prendere allora in conto esclusivamente le differenze costitutive della capacità di formare e di definire l'identità individuale e culturale, ma sulla base di quali criteri ? Come rispettare diritti fondamentali, universali, quali quelli della libertà, della giustizia e dell'uguaglianza? (Bell 1993).

A questo punto la riflessione filosofica e politica ha preso il posto che gli studi antropologici e sociologici hanno avuto lungo tutto il secolo $\mathrm{XX}^{\circ}$ nelle ricerche sulla cultura.

\section{BIBLIOGRAFIA}

ABOU, S., L'identité culturelle. Relations interethnique et problèmes d'acculturation, Paris 1981.

- Cultures et droits de l'homme, Paris 1992.

AMSELLE, J.-L., Logiques métisses. Anthropologie de l'identité en Afrique et ailleurs, Paris 1990.

- Vers un multiculturalisme français. L'empire de la coutume, Paris 1996.

APOSTEL, L., Le né-connexionisme et la théorie de l'argumentation, in Pensée naturelle, logique et Langage. Hommage à Jean-Bl.aise Grize, Neuchâtel 1987, pp. 31-45.

ARCHER, M., Culture and Agency. The Place of Culture in Social Theory, Cambridge, Mass., 1988.

BADIE, B., Culture et politique, Paris 1983.

BAUDRILLARD, J., De la séduction, Paris 1979.

- Simulacres et simulation, Paris 1981.

- Le ludique et le policier \& autres écrits parus dans «Utopie» (1967-1978), Paris 2001.

BELL, D. A., Communitarism and its Critics, Oxford 1993.

BECK, U., Nicht Autonomie, sondern Bastelbiographie, «Zeitschrift für Soziologie», 22, 3, June 1993, pp. 178-197.

BENETTON, Histoire de mots. Culture et Civilisation, Paris 1975. 
BERLIN, I., Le bois tordu de l'humanité, Paris 1992.

BERNSTEIN, R., Dictatorship of Virtue: Multiculturalism and the Battle for America's Future, New York 1994.

BISSOONDATH, N., The Seven Illusions. Multiculturalism in Canada, Toronto 1994.

BOUDON, R., The Origin of Values, New Brunswick/London 2001.

BUSINO, G., Quelles significations attribuer aux processus de rationalisation de la mondialisation?, in MERCURE, D.(dir.), Une société monde? Les dynamiques sociales de la mondialisation, Saint-Nicolas,Québec, 2001,pp. 167-181.

- Il dibattito sulla scienza nelle ricerche recenti. Dal «Programma forte» alle controversie dell'«affare Sokal», in «Rivista storica italiana », CXI, 1999, fasc.III, pp. 706-755.

CAMPBELL, B., Goliath, Britain's Dangerous Place, Methuen 1995.

CERTEAU, M. de, La culture au pluriel, Paris 1974 (ed. riv. Paris 1993)..

- L'invention du quotidien, Paris 1980.

CHAUMONT, J.-M., Notes sur la tradition communautarien, Louvain-la-Neuve 1990.

CLIFFORD, J., Malaise dans la culture. L'ethnographie, la littérature et l'art au XXe siècle, Paris 1996 (Ia ed.in inglese 1988).

COLE, L., SCRIBNER, S., Culture and Tought : a Psychological Introduction, New York 1974.

COULDRY, N., Inside Culture, Re-Imagining the Method of Cultural Studies, London 2000.

COUCHE, D., La notion de culture dans les sciences sociales, Paris 1996.

DOUGLAS, M., NEY, S., Missing Persons : a Critique of Personehood in the Social Sciences, London 1998.

DWORKIN, R., A matter of Principle, Cambridge, Mass. 1985.

FEATHERSTONE, M., Global Culture. Nationalism, Globalization and Modernity, London 1990.

GALSTON, W.A., Justice and the Human Good, Chicago, Mass. 1980.

GEERTZ, C., Thick Description : Toward an Interpretative Theory of Culture, in The Interpretation of Cultures: Selected Essays, 3-30, New York 1973.

- Liberal Purpose: Goods, Virtues, and Diversity in the Liberal State, New York 1991.

GIDDENS, A., Runaway World, London 1999.

GLAZER, N., We Are All Multiculturalists Now, Cambridge, Mass., 1997.

GLICK SHILLER, N., BASCH, L., BLANC-SZANTON, C. Towards a Transnational Perspective on Migration. Race, Class, Ethnicity, and Nationalism Reconsidered, New York 1992.

GRAY, J., Two Faces of Liberalism, London 2001.

GRIGNON, C. \& PASSERON, J.-C., Le savant e le populaire. Misérabilisme et populisme en sociologie et littérature, Paris 1989.

HANNERZ, U., Transnational Connection. Culture, People, Places, London 1997.

HEELAS, P., LASH, S., MORRIS P. ( eds.), Detraditionalization : Critical reflections on Autority and Identity, Oxford 1995.

HOLMES, S.,The Anatomy of Antiliberalism, Cambridge, Mass., 1993.

HOGGART, R., The Use of Literarcy, London 1957.

- A Local Habitation, London 1988.

IACOVETTA, F., PERRIN, R., PRINCIPE, A., Enemies Within, Toronto 2000.

JAMESON, F., Postmodernism or, the Cultural Logic of Late Capitalism, Durham 1991.

KAUTZ, S., Liberalism, Community, Ithaca, NY 1997.

KEKES, J., The morality of Pluralism, Princeton, NJ, 1993.

KROEBER, A. L., KLUCKHOHN, C., Culture. A critical Review of Concepts and Definitions, Cambridge, Mass., 1952 ( tr. it.: Il concetto di cultura, Bologna 1972).

KUPER, A., Culture: the Anthropologist Account,

KYMLICKA, W., Liberalism, Community and Culture, Oxford 1993. 
- Multicultural Citizenship : A Liberal Theory of Minority Groups, Oxford 1995.

- $\quad$ and NORMAN, W., (eds), Citizenship in Diverse Societies, Oxford 2000.

- Politics in the Vernacular : Nationalism, Multiculturalism and Citizenship, Oxford 2001.

LASCH, C., Mass Culture Reconsidered?, New York 1981

- - The Revolt of the Elites and the Betrayal of Democracy, New York 1996.

LUKES, S., Different Cultures, Different Rationalities, «History of the Human Sciences», 13, 1, 2000, pp. 3-18.

LE THANK KHOI, Culture, créativité et développement, Paris 1992.

LEVI-STRAUSS, C., Race et culture, «Revue internationale des sciences sociales », $\mathrm{n}^{\circ} 4,1971$, pp. 647-666.

- Culture et nature. La condition humaine à la lumière de l'anthropologie, «Commentaire », $\mathrm{n}^{\circ} 15$, 1981, pp. 365-372.

MAC INTYRE, A., After Virtue : A Study in Moral Theory, London 1981.

MC ROBBIE, A., Feminism and Youth Culture, London 1991.

MARRUS, H.-I, Culture, civilisation, décadence, «Revue de synthèse », décembre 1938.

MATTELART, A. \& NEVEU, E., «Cultural Studies'Stories». La domestication d'une pensée sauvage?, «Réseaux», 1996, n 80, pp. 12-58.

MURDOCK, G. P., Social structure, New York 1949 ( tr. it. La struttura sociale, Milano 1971)

- Culture and Society, Pittsburg 1965.

MORENO, D. \& QUAINI, M., Per una storia della cultura materiale, «Quaderni storici», 1976, n. 31, pp. 5-37.

OLDFIELD, A., Citizenship and Community: Civic Republicanism and the Modern World, London 1990.

PENDAKUR, R., Immigrants and the Labour Force. Policy, Regulation and Impact, Montreal 2000.

POWER, A., Estates on the Edge. The Social Consequences of Mass Housing in Northern Europe, New York 1997.

POUTIGNAT, Ph. \& STREIFF-FENART, Théories de l'ethnicité, Paris 1995.

PUTNAM, R., LEONARDI, R., NANETTI, R. Y., Making Democracy Work: Civic Traditions in Modern Italy, Princeton, NJ, 1993.

PUTNAM, R., Bowling Alone. The Collapse and Revival of American Community, New York 2000.

RAWLS, J., Political Liberalism, New York 1993.

ROSZAK, T., The Making of a Counter Culture, New York 1969.

SABETTI, F., The Search for Good Government, Montreal 2000.

SANDEL, M., Liberalism and the Limits of Justice, New York 1992.

SCHNAPPER, D., La communauté des citoyens. Sur l'idée moderne de nation, Paris 1994.

- La relation à l'autre. Au cœur de la pensé sociologique, Paris 1998.

SEMPRINI, A., Le multiculturalisme, Paris 1997.

SPIRO, M. E., Culture and Human Nature, Chicago, Mass., 1987.

STAROBINSKI, J., Le remède dans le mal. Critique et légitimation de l'artifice à l'âge des Lumière, Paris 1989.

STOREY, J., Cultural Theory and Popular Culture, Glencoe, Ill., 2001.

TAYLOR, C., Sources of the Self. The Making of the Modern Identity, Cambridge, Mass. 1989.

- Shared and Divergent Values, in WATTS, R.L. \& BROWN, D.M. (eds), Options for a New Canada, Toronto 1991, pp. 53-76.

- $\quad$ The Malaise of Modernity, Concorde 1991.

- Multiculturalism and «The Politics of Recognition», Princeton, NJ, 1992.

- Philosophical Arguments, Cambridge, Mass. 1992. 
- The Politics of Recognition, in GUTMANN, A. (ed.), Multiculturalism. Examining the Politics of Recognition, Princeton, NJ, 1994, pp. 25-74..

- Why Democracy Needs Patriotism?, in COHEN, J. (ed.), For Love of Country. Debatting the Limits of Patriotism. Martha C. Nussbaum with Respondents, Boston, Mass., 1996.

- La liberté des modernes, Paris 1997.

THOMAS, N., Colonialism's Culture: Anthropology, Travel and Government, Princeton, NJ, 1994. TURNER, B. (ed.), Theories of Modernity and Post-Modernity, London 1990.

WALZER, M., Radical Principles. Reflections of An Unreconstructed Democrat, New York 1980.

- Spheres of Justice: A Defense of Pluralism and Equality, Cambridge, Mass. 1983.

- Pluralism: A Political Perspective, KYMLICKA, W. (ed.), The Rights of Minority Culture, New York 1995, pp. 139-154.

WARNIER, J.-P., La mondialisation de la culture, Paris 1999.

- Construire la culture matérielle. L'homme qui pensait avec ses doigts, Paris 1999.

WILLIAMS, R., Problems in Materialism and Culture: Selected Essays, London 1980.

WILLIS, P., Common Culture, Open University, 1990.

WILSON, B., Morality in the Evolution of the modern social System, «British Journal of Sociology », 36, 3, September 1985, pp. 315-332. 